\title{
Inserção de Agilidade nos Processos de Teste de uma Organização de Software MPS.BR: Um relato de experiência
}

\section{Ciro Grippi Barbosa Lima}

Universidade Federal do Rio de Janeiro - COPPE/Sistemas Caixa Postal 68.511 - CEP

21.941-972 - Rio de Janeiro - RJ - Brasil

\{cblima,ght\}@eos.ufrj.br

\begin{abstract}
Software testing processes are usually affected by requirements and risks changing requirements throughout the software project. Changes must be done with flexibility and agility without disrupting effort, test coverage and software quality. Therefore, this paper describes our experience on introducing agility in software testing processes into a MPS.BR organization level C. The preliminary results indicate that using appropriate standards for the construction of Test Cases and Scripts promotes reuse, and increasing the project visibility improves process understanding between development and testing teams.
\end{abstract}

Resumo. Processos de testes de software são usualmente afetados pela necessidade de pronto atendimento a mudanças de requisitos e sua adequação ao conjunto de riscos do projeto. Atender a estas mudanças com flexibilidade e agilidade, sem afetar o esforço, cobertura de testes e a qualidade final do produto é um desafio. Assim, relatamos nesse trabalho nossa experiência na introdução de agilidade em processos de testes de software em uma organização nível C MPS.BR. Os resultados obtidos até o momento indicam que o uso de padrões adequados para construção dos Casos e Roteiros de Teste promove seu reuso, e o aumento de visibilidade do projeto melhora o entendimento do processo entre as equipes de desenvolvimento e testes.

\section{Introdução}

No ambiente de negócios da indústria, a busca por eficiência nos processos de software está inserida em um contexto no qual ocorrem mudanças frequentes de prioridades, alteração do escopo de projetos e, demanda crescente por qualidade. Sob a perspectiva da gestão das atividades de testes de software, dotar um processo de testes de características que facilitem a adaptação rápida às mudanças, promova a cooperação entre os stakeholders, e antecipem as atividades de planejamento de testes pode trazer ganhos na produtividade da equipe envolvida, e representa um desafio de pesquisa.

A especificação IEEE-829/2008 cobre o ciclo de vida das atividades de testes e prevê o atendimento a estas questões ao incluir uma atividade específica de identificação e revisão do nível de integridade em seus processos. Nesta atividade o Gerente de Testes pode avaliar conjuntamente com os stakeholders as consequências da ocorrência de eventuais falhas, determinando assim a gravidade associada e as necessidades de mudança no processo.

Considerando este cenário, Abrantes [2012] conduziu um grupo de estudos sobre agilidade em processos de software identificando um conjunto de dezesseis 
características $^{1}$ e quinze práticas ${ }^{2}$. Tomando por base estas características e práticas de agilidade e um processo de teste de software [Dias Neto, 2006] inspirado na IEEE-829 foram realizados dois mapeamentos: (a) entre características e práticas, identificando que práticas poderiam contribuir para caracterizar um processo com agilidade e, (b) entre práticas e atividades de testes. Estes resultados estabeleceram hipóteses para futura investigação.

Em continuidade, os autores desse artigo iniciaram estudo visando explicitar evidência sobre a presença das características de agilidade em processos de teste de software, identificando, por exemplo, que práticas adicionais e relacionadas ao "Projeto de Procedimentos e Casos de Testes (CTs)" poderiam permitir aprimorar a manutenibilidade, capacidade para apoiar validação de requisitos e automação para facilitar a execução de testes de regressão. A manutenção de uma suíte de CTs atualizada em que os testes possuem rastreabilidade bidirecional com os requisitos apoiando a determinação dos impactos decorrentes de mudanças de escopo também se mostrou necessária [Cornelissen et al, 2009]. Na atividade de execução dos testes foram destacados aspectos de integração entre desenvolvimento e teste, automação de execução [Stolberg, 2009] e a adoção da abordagem test-first [Vu et al, 2009]. Entretanto, chama atenção o fato de que, apesar dos trabalhos mencionarem atividades de teste utilizadas na indústria com resultados positivos, em nenhum deles foi mencionada a existência de um processo de testes previamente definido. Por exemplo, a necessidade de formalização dos critérios, atividades e estratégias de teste aparece explicitamente em estudo [Hanssen and Haugset, 2008] sobre adoção de uma estratégia baseada em testes de aceitação automatizados. A falta de critérios e procedimentos na definição de cobertura dos CTs representou uma das experiências negativas relatadas.

A adoção de processos de software definidos é uma prática comum em organizações que adotam modelos de maturidade, por exemplo, o MPS-BR. Desta forma, estes resultados combinados com a disponibilidade de processos nos motivaram a planejar e iniciar a execução de um estudo de caso na indústria para observar as relações entre práticas e características de agilidade nos processos de teste estabelecidos e em uso em uma organização de software. Este relato descreve a execução deste estudo e apresenta alguns resultados preliminares obtidos através de procedimentos para apoiar a melhoria das atividades de testes, alcançados através da inserção das práticas Visibilidade de Projeto e Design Simples. Assim, na seção 2 são apresentadas a contextualização, caracterização e planejamento do estudo. A seção 3 descreve a execução e os resultados obtidos até o presente momento. E por fim a seção 4 contém as considerações finais e lições aprendidas.

\section{Caracterização e Planejamento}

$\mathrm{Na}$ definição dos procedimentos de trabalho foi adotada a abordagem definida no paradigma de melhoria contínua [Basili et al, 1992] composta das etapas caracterização, planejamento, execução e empacotamento.

\footnotetext{
${ }^{1}$ Adaptabilidade, Auto-organização, Emergência, Equipes Pequenas, Incorporação de Retroalimentação, Leanness, Modularidade, Orientação a Pessoas, Reflexão e Introspecção, Ser Colaborativo, Ser Cooperativo, Ser Incremental, Ser Iterativo, Testes Constantes, Time-Boxing e Transparência.

${ }^{2}$ Backlog de produto, Cliente Presente, Desenvolvimento Orientado a Testes, Design Simples, Equipe Completa, Integração Contínua, Jogo de Planejamento, Metáfora, Padrões de Código, Propriedade Coletiva do Código, Refatoração, Liberações Frequentes, Reuniões Diárias, Ritmo Sustentável, Visibilidade de Projeto.
} 


\subsection{Caracterização}

A organização selecionada executa um programa permanente de melhoria em seus processos de software acompanhado por um cronograma de atividades organizacionais e pelo grupo de processos SEPG (Software Engineering Process Group). É nível C MPS.BR [SOFTEX, 2011] e segue a ISO 9001 [ISO/IEC,2008]. Atualmente seu processo de desenvolvimento possui 74 atividades dispostas em 3 fases: Especificação e Planejamento de Projeto, Análise Projeto e Construção de Software e Homologação e Implantação. Práticas Scrum são adotadas para apoiar a gerência de projetos. A equipe de desenvolvimento conta com 14 profissionais envolvidos nos processos de software, tendo dois analistas de teste e dois testadores.

Nas entrevistas com a equipe de testes os pontos mais relevantes identificados foram: (a) testes funcionais são os mais utilizados (testes de integração cuidam mais da preparação do ambiente para testes). A ferramenta TestLink é adotada para o projeto dos CTs; (b) O gerente define quais CTs serão utilizados para teste de regressão a partir do relacionamento dos requisitos e Casos de Uso (UCs) aos CTs, não sendo utilizado critério de cobertura; (c) os testes não são automatizados; (d) Os CTs são projetados ad-hoc, e; (e) por vezes passos de CTs já construídos anteriormente são novamente escritos, quando ocorrem pequenas mudanças, gerando retrabalho.

A análise dos artefatos de testes foi executada para três grandes projetos mantidos pela organização, focando na ferramenta para Gerência e Acompanhamento de Projetos apresentando 5330 CTs (acumulados desde março de 2008) com seus releases mantidos para diferentes clientes. As atividades de Verificação, Validação e Testes estão definidas conjuntamente com as de desenvolvimento em seu processo padrão disposto em uma instrução de procedimento, mantida em único documento.

Com o propósito de facilitar a compreensão dos processos, fornecer diferentes níveis de apoio à equipe, e refletir rapidamente mudanças, de acordo com a prática "Visibilidade de Projeto" foi adotada uma linguagem de modelagem de processos (PML - Process Modeling Language) para documentação. Estes pré-requisitos convergem com algumas diretrizes sugeridas para gestão de conhecimento dos processos, na qual os modelos não servem apenas como meio de armazenamento sobre melhores práticas, mas também provêm links para itens de conhecimento [Holz et al, 2001].

Para esta escolha foi acordado o uso do SPEM [OMG, 2004] e do framework EPF Composer, pois uma das principais premissas era selecionar uma linguagem capaz de promover um entendimento comum das atividades de teste entre os participantes, sem que fosse necessária a simulação e execução dos processos, ponto forte do BPMN [OMG, 2010]. Além destes aspectos, a existência de relatos de uso do SPEM [Ribeiro et al, 2011] para apoiar a reutilização de elementos de um processo de software direcionaram esta escolha. O SPEM foi utilizado para documentar o processo de software da organização e o processo de teste proposto como padrão, e também os procedimentos propostos para inserção de agilidade [INSERCAOAGIL].

\subsection{Planejamento}

A documentação do processo de testes padrão considerou as informações de atividades, perfis e artefatos descritas em [Dias Neto, 2006] contemplando: Planejar Teste, Projetar Teste, Especificar CT, Definir Procedimentos de Teste, Executar Testes, Analisar Resultados - acrescidas da atividade de monitoramento, controle e replanejamento 
[TESTE_IEEE829]. A documentação das atividades da organização abrangeu testes e desenvolvimento e foi publicada em sua intranet [TESTE_ORG].

Antes de avaliar possibilidades de inserção de agilidade foi realizada uma comparação entre as atividades da organização e aquelas previstas no processo de teste padrão para verificar se: (a) determinada atividade está presente em apenas um dos processos, (b) as atividades estão modeladas em ambos os processos, porém executadas em etapas distintas, (c) apresentam o mesmo objetivo, porém contendo artefatos diferentes ou subatividades sem similaridade. Um dos objetivos deste mapeamento foi avaliar a existência de eventuais gaps. Assim, foi prevista a possibilidade de inclusão de uma determinada atividade nos processos da organização. Apesar de aparentar um contra-senso, a inserção de atividades ou seu rearranjo podem trazer agilidade aos trabalhos. Para esta comparação foi utilizada uma planilha entregue em 05/08/2012 para o SEPG, em que foram dispostas lado-a-lado, as atividades de ambos os processos.

Ao realizar a comparação entre as atividades de planejamento foi constatado que o processo da organização não possuía uma atividade de caracterização de testes em sua fase de Especificação e Planejamento do Projeto (Item 1 Tabela 1). Na reunião com o SEPG foi ponderado que a caracterização deveria ser incluída após a atividade "Avaliar e Aprovar os Requisitos e Arquitetura de Alto Nível", pois antes disso, o conhecimento sobre os requisitos está restrito às informações do termo de abertura. Feito antes poderia limitar a efetividade da caracterização dos testes.

Tabela 1 - Relação de mudanças/inclusões sugeridas para as atividades

\begin{tabular}{|c|c|c|c|}
\hline $\begin{array}{l}\text { Macro } \\
\text { Atividade }\end{array}$ & Atividade(s) & Diferenças apontadas & Observações \\
\hline $\begin{array}{l}\text { 1-Planejar } \\
\text { Testes }\end{array}$ & $\begin{array}{l}\text { "Caracterizar } \\
\text { Testes" e "Planejar } \\
\text { Estratégia". }\end{array}$ & $\begin{array}{l}\text { Não estão definidas } \\
\begin{array}{l}\text { Processo no } \\
\text { organização. }\end{array}\end{array}$ & $\begin{array}{l}\text { Pode subsidiar uma melhor estimativa de } \\
\text { esforço do teste e mitigar os riscos de } \\
\text { forma pró-ativa. }\end{array}$ \\
\hline $\begin{array}{l}\text { 2-Projetar } \\
\text { Testes }\end{array}$ & $\begin{array}{l}\text { "Identificar Casos e } \\
\text { Procedimentos de } \\
\text { Testes" }\end{array}$ & $\begin{array}{l}\text { EspecificarCT e Identificar } \\
\text { Casos e Procedimentos de } \\
\text { Teste são realizadas em uma } \\
\text { única etapa. }\end{array}$ & $\begin{array}{l}\text { Realizar as tarefas em duas etapas pode } \\
\text { apoiar a padronização de Projeto de } \\
\text { Caso de Teste. }\end{array}$ \\
\hline
\end{tabular}

Em relação ao projeto de testes (Item 2 Tabela 1), o SEPG questionou a necessidade de existirem atividades específicas para identificação e especificação dos CTs, pois apesar de não estar explícito, o analista de teste é orientado a cada CT, definir a entrada, os resultados esperados e procedimentos, para só então descrevê-los. Após esclarecimentos sobre a importância desta especificação - desde que incentiva a adoção de melhores práticas, apoiando separação entre dados e passos - esta sugestão foi aceita.

Nas diferenças apontadas pela comparação entre os artefatos de cada processo foram priorizadas aquelas associadas às mudanças nas atividades de projeto de CTs. Com isto o plano de teste passou a conter uma seção na qual são caracterizadas as funcionalidades a serem testadas, definindo prioridades e riscos associados, e outra seção em que os procedimentos e CTs são identificados antes da análise e construção.

Após a definição dos ajustes nestas atividades foram avaliadas as possibilidades de inserção das práticas de agilidade e criada uma matriz baseada no mapeamento sugerido por Abrantes (2012). Nesta avaliação foi constatado que em alguns casos a prática já era utilizada parcialmente ou em toda sua abrangência, decorrente da adoção do método Scrum (práticas "Reuniões Diárias" e "Liberações Frequentes" na Figura 1 com um símbolo check). Em outros, as atividades de testes não utilizavam a prática avaliada, contudo, por conta de certas características da empresa não era aplicável ou de 
difícil adoção (aparecem na Figura 1 com um circulo vazio). Isto aconteceu, por exemplo, em relação à prática "Metáfora" capaz de apoiar o entendimento de novos domínios - sendo que a organização trabalha com domínios bem definidos e estáveis.

\begin{tabular}{|l|l|l|l|l|l|l|l|l|c|}
\hline Latividades & $\begin{array}{l}\text { Reuniôes } \\
\text { Diáras }\end{array}$ & $\begin{array}{l}\text { Liberacōes } \\
\text { Frequentes }\end{array}$ & Metáfora & $\begin{array}{l}\text { Equipe } \\
\text { Completa }\end{array}$ & $\begin{array}{l}\text { Cliente } \\
\text { Presente }\end{array}$ & $\begin{array}{l}\text { Design } \\
\text { Simples }\end{array}$ & $\begin{array}{l}\text { Visibilida } \\
\text { de } \\
\text { Projeto }\end{array}$ & $\begin{array}{l}\text { Backlog } \\
\text { de } \\
\text { Produto }\end{array}$ & $\begin{array}{l}\text { Jogo de } \\
\text { Planejamento }\end{array}$ \\
\hline Planejar Testes & $\checkmark$ & & 0 & & 0 & & $\bullet$ & $\bullet$ & $\bullet$ \\
\hline Projetar Testes & $\checkmark$ & & 0 & 0 & 0 & $\bullet$ & $\bullet$ & & $\bullet$ \\
\hline Especificar CTs & $\checkmark$ & & & 0 & & $\bullet$ & $\bullet$ & & \\
\hline $\begin{array}{l}\text { Definir } \\
\text { Procedimentos }\end{array}$ & $\checkmark$ & & & & & $\bullet$ & $\bullet$ & & \\
\hline Executar Testes & $\checkmark$ & $\checkmark$ & & & & & $\bullet$ & & \\
\hline Analisar Resultados & $\checkmark$ & $\checkmark$ & & & & & $\bullet$ & & \\
\hline $\begin{array}{l}\text { Monitorar } e \\
\text { Controlar } 0 \\
\text { Processo de Teste }\end{array}$ & $\checkmark$ & $\checkmark$ & & & & & $\bullet$ & & \\
\hline
\end{tabular}

Figura 1 - Avaliação de práticas de agilidade a serem utilizadas no Processo de Teste

Quanto à adoção da prática "Equipe Completa" um dos participantes ponderou que em um dos projetos foi sentida a falta de um especialista em segurança, porém restrições de orçamento impediram sua contratação. Por último, foram classificadas as associações passíveis de serem implementadas (aparecem c/ um círculo preenchido).

Ao avaliar as atividades de design de CT e a forma como os artefatos eram construídos foi observada a falta de aderência a um padrão. Em muitos CTs as entradas e pré-condições estavam descritas lado-a-lado na seção dos passos de cada procedimento (Item 1 Figura 2). O que parece ser uma consequência da ausência de uma atividade de projeto na qual, inicialmente, os CTs são identificados, depois os procedimentos, para na sequência serem desenvolvidas as entradas, pré-condições, resultados esperados. $\mathrm{O}$ mesmo $\mathrm{CT}$ foi reescrito levando em conta as boas práticas de projeto ficando a responsabilidade de cada elemento bem-definida (Item 2 Figura 2).

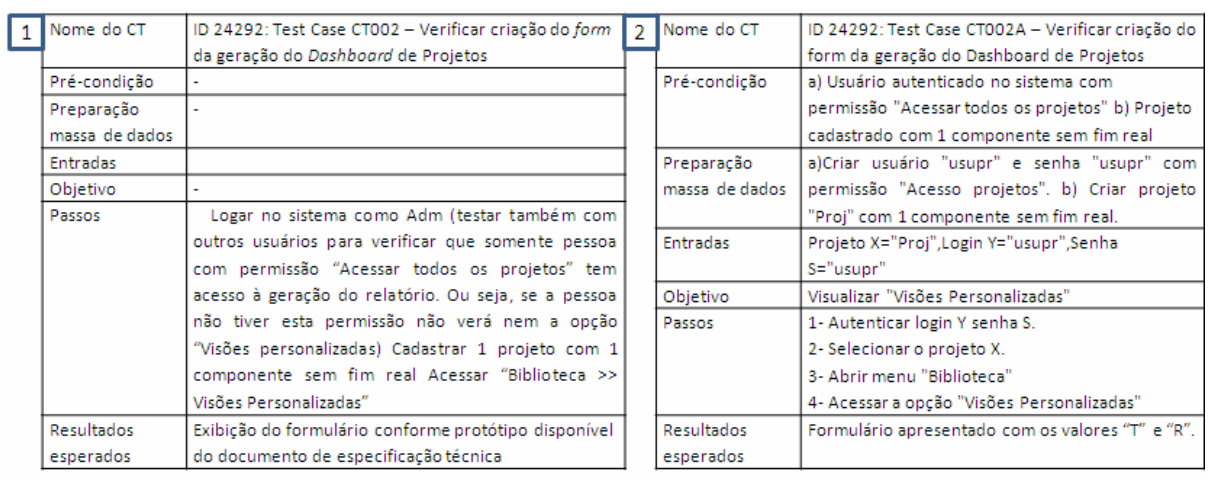

Figura 2 - Exemplo de CT de releases anteriores e CT com adoção de melhores práticas

Ao realizar o mesmo trabalho para uma sequência de CTs percebeu-se um aumento considerável no número destes artefatos, pois nas versões originais eram dispostos mais de um valor para entrada e resultados esperados. Conjuntamente com estas descobertas também foi diagnosticada a necessidade de facilitar o acesso e a recuperação dos CTs projetados em releases anteriores.

A adoção da prática "Visibilidade de Projeto" através da qual é ratificada a importância da equipe estar informada constantemente de mudanças no projeto e nos modelos pode aumentar a incidência de reuso e minimizar retrabalho decorrente da reescrita de procedimentos. Ao adotar as premissas (mitigação de complexidade desnecessária e código extra) de "Design Simples" é esperada uma maior facilidade na identificação de procedimentos e CTs apoiando a manutenção decorrente de mudança 
de requisitos. Considerando estas expectativas e as oportunidades de observação, foi priorizada a adoção destas duas práticas em reunião em 19/10/2012, sendo gerada uma nova versão do processo na intranet da organização [TESTE_AGIL].

A questão de pesquisa foi elaborada com o objetivo de investigar se uma equipe de testes executando um conjunto de atividades responsáveis pela caracterização, identificação e projeto de procedimentos e CTs e adotando um padrão (template) tende a reutilizar uma quantidade maior destes artefatos, do que equipes executando um processo no qual estas atividades não foram prescritas. Portanto, a quantidade de CTs reutilizados foi utilizada como surrogate para observar as práticas "Visibilidade de Projeto" e "Design simples". O planejamento do estudo foi concluído em 12/11/2012.

No levantamento de alternativas de fornecimento de material de suporte que favorecesse a visualização dos CTs e apoiasse a adoção de um padrão foi considerado o uso de algumas ferramentas open-source disponíveis. Contudo, a oportunidade de desenvolvê-los atendendo às perspectivas e requisitos específicos do estudo de caso, e o contexto das atividades de teste da organização incentivou a condução de um projeto de um editor. $\mathrm{Na}$ elicitação dos requisitos foi adotado como premissa desenvolver um produto capaz de facilitar a edição dos blocos de cada procedimento dos CTs.

A expectativa é que através do uso contínuo, a organização passe a contar com uma biblioteca de componentes de procedimentos e CTs recuperáveis a partir de um conjunto de palavras-chave. Para isto foi desenvolvido o conceito de actionList (ACL). Uma actionList é um componente constituído de um conjunto de ações previamente cadastradas que poderão ser associadas pelo analista de teste a um passo de um procedimento, pré-condição, ou verificação de resultado esperado. Foi também previsto no modelo a possibilidade de uma ACL estar associada a um comando $S d k$ de motor de testes automatizados. O editor ficou disponível em 12/12/2012 com uma lista de funções para projeto de CTs, consulta matriz de rastreabilidade bidirecional, cadastro de projetos, releases, requisitos, UCs, cenários e relatório de progresso dos testes.

\section{Execução e Resultados Obtidos.}

A organização reservou dois sprints para manutenção de funcionalidades da ferramenta de GP, utilizando uma instância do novo processo, o template e o editor. O objetivo foi avaliar o nível de reuso de CTs desenvolvidos anteriormente e transcritos para o novo padrão. Contudo, como o cliente priorizou um novo módulo a oportunidade de observação do nível de reuso abrangeu o desenvolvimento de todo este módulo.

Em 02/01/2013 foram treinados dois analistas, um com mais de cinco anos de experiência em testes, outro com um ano de experiência e dois testadores iniciantes.

Foram gerados cento e vinte e dois CTs para atender dez cenários de execução mapeados, associados a onze procedimentos (Figura 3).

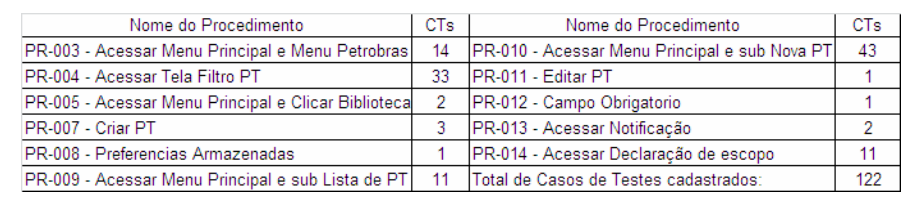

Figura 3 - Relação entre procedimentos e Casos de Teste desenvolvidos

A caracterização de testes iniciou em 04/01/2013, porém por conta de mudanças de prioridade do cliente, o projeto foi adiado e a análise e construção dos CTs iniciaram 
apenas em 01/03/2013. O trabalho foi realizado pelo analista de menor experiência, consumindo 38 horas de esforço. Um mesmo componente responsável pela verificação de perfil de usuário foi associado a cada pré-condição de oitenta e cinco CTs. Na Figura 4 é exibido um CT que utilizou esta ACL, e o recurso de auto-complete na associação a uma rotina de resultado esperado.

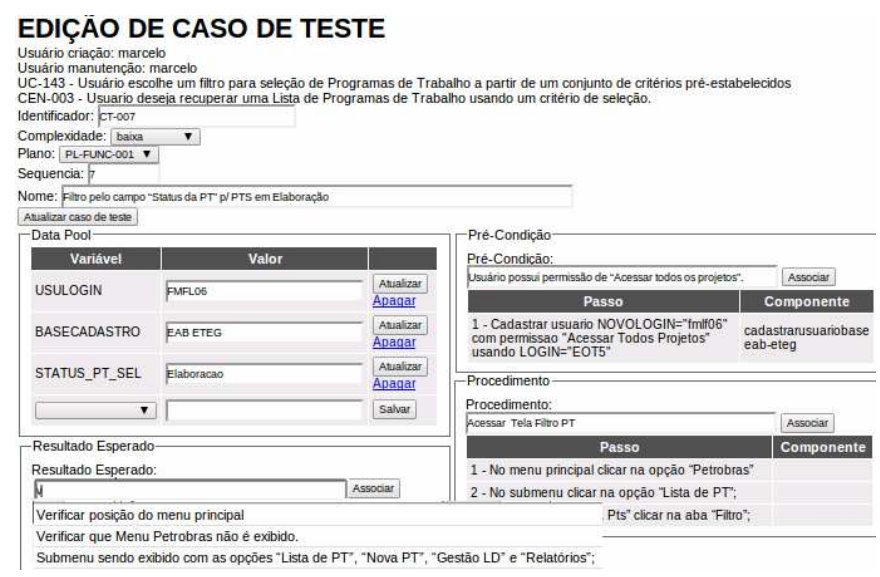

Figura 4 - Exemplo de Caso de Teste desenvolvido a partir do Editor

Na Figura 5 são mostrados os passos do componente.
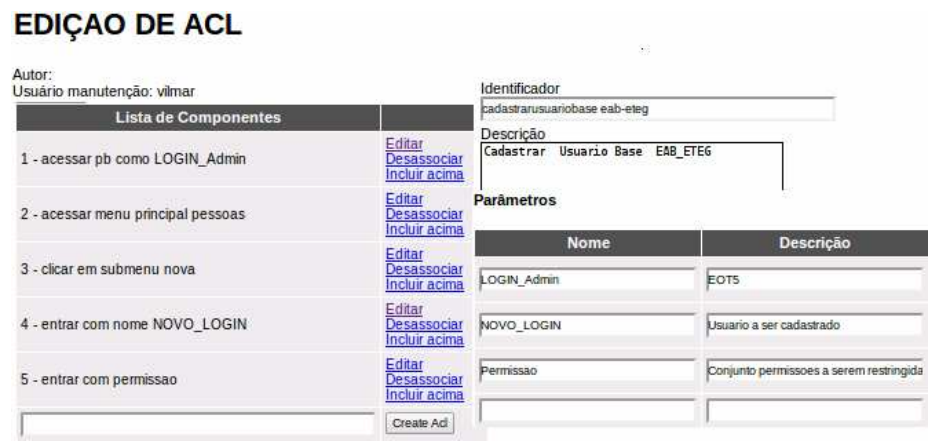

Figura 5 - Exemplo de Componente a ser reutilizado

Durante a escrita deste artigo foi iniciada a execução e coleta de esforço dos testes. Quanto às mudanças no processo, a equipe passou uma avaliação positiva da inclusão de uma atividade de caracterização de testes - quando os CTs e procedimentos foram identificados no início do projeto e classificados por impacto e risco associado ao negócio. A organização decidiu adotar o editor nos próximos releases do projeto, e o EPF para manutenção dos processos. Após encerramento do release serão avaliadas as hipóteses do estudo, e na sequência realizado o empacotamento.

\section{Considerações Finais.}

Este trabalho relatou a experiência de inserção de práticas e características de agilidade em um processo de teste. Na etapa de caracterização foi possível observar um conjunto de melhorias priorizadas e implementadas conjuntamente com a organização, objetivando o aumento de eficiência através da mitigação de retrabalho e sobreposição de CTs. Segundo os participantes que utilizaram o template e o processo modificado, a separação dos elementos de cada CT e a possibilidade de utilizá-los a partir de uma interface facilitou a análise e construção, não sendo mais necessário associar mais de 
um par de entradas e resultado esperado no cadastro de um CT. Também relataram que o uso de componentes permitiu encapsular as sequências de passos de navegação entre as telas do software sob teste. Anteriormente elas eram copiadas e repetidas em cada CT. Segundo um analista que participou do estudo, os mapas de rastreabilidade e o uso de cenários facilitaram a organização e recuperação dos CTs. O encerramento da coleta de dados e sua consolidação estão previstos para as próximas semanas agregando mais informações aos resultados obtidos até o presente momento.

\section{Agradecimentos}

Agradecemos a Marcelo S. de Mello (responsável pelo SEPG), aos diretores Eduardo C. Carvalho e José Vilmar E. de Souza da Informal Informática e a Neilson Carvalho pelo apoio na construção do Editor de CTs. Prof. Travasssos é pesquisador apoiado pelo CNPq.

\section{Bibliografia}

Abrantes J. F. (2012) "Práticas e Características de Agilidade em Processos de Teste de Software", In COPPE/UFRJ Tese de Doutorado, 2012.

Basili, V., Caldiera, G., Mcgarry, F., Pajersky, R., Page, G., Waligora, S., (1992) The Software Engineering Laboratory an operational software experience factory, ACM, 1992

Dias Neto, A. C. (2006) "Uma Infraestrutura Computacional para apoiar o planejamento e controle de testes de software" Dissertação M. Sc., COPPE/UFRJ, 2006.

Hanssen, G. K., Haugset, B. (2008) "Automated Acceptance Testing-A Literature Review and an Industrial Case Study" In Agile Conference 2008.

Holz, H., Könnecker, A., Maurer, F. (2001) "Task-Specific Knowledge management in a Process-Centered SEE”, K.-D. Althoff, Feldmann, and W. Müller (Eds.): LSO 2001, LNCS 2176, pp. 163-177.

IEEE Std 829тм-2008 (Revision of IEEE Std 829-1998).

INSERCAOAGIL. Procedimentos para Inserção de Agilidade em Processos de Testes. Disponível em http://lens-ese.cos.ufrj.br/insercaoagil/. Acesso em: 13/05/13.

Cornelissen, B., Van Deursen, A., Moonen, L., Zaidman, A. (2007) "Visualizing Test Suites to aid in software understanding" In 11th European Conference on Software Maintenance and Reengineering, 2007.

NBR/ISO, 2008. ABNT - Sistemas de Gestão da Qualidade.

OMG (2004) "Software Process Engineering Meta-model (SPEM) 2.0" RFP, Nov. 2004

OMG (2010) "Business Process Model and Notation (BPMN), Version 2.0 (2010)"

Ribeiro, L. C. M., Ramos, C. S., Crozara, K., Neri, H. R., Alves E., Figueiredo, R. M. C. (2011) "Definição de Processos de Software baseada em uma Arquitetura de Componentes de Processo". In SBQS 2011

SOFTEX, 2011. "MPS.BR: Melhoria de Processo do Software Brasileiro, Guia Geral.

Stolberg, S. (2009) "Enabling Agile Testing through Continuous Integration", In Agile Conference, 2009.

TESTE_AGIL. Processo de Projeto e Desenvolvimento com propostas de mudanças. Disponível em http://lens-ese.cos.ufrj.br/it7 3 pro/. Acesso em: 13/05/13.

TESTE_IEEE829 - Processo de Teste Baseado IEEE-829. Disponível em http://lensese.cos.ufrj.br/teste IEEE829/index.htm. Acesso em: 13/05/13.

TESTE_MPSBR. Processo Padrão de Projeto e Desenvolvimento. Disponível em http://lens-ese.cos.ufrj.br/it7 3/index.htm. Acesso em: 13/05/13.

Vu, J.H., Frojd, N., Shenkel-Therolf, C., Janzen, D.S. (2009) "Evaluating test-driven development in an industry-sponsored capstone project" In Conference of $6^{\text {th }}$ International Conference on Information Technology: New Generations, ITNG, 2009 